\title{
Low Level Laser Therapy With an 810-nm Diode Laser Affects the Proliferation and Differentiation of Premature Osteoblasts and Human Gingival Fibroblasts In Vitro
}

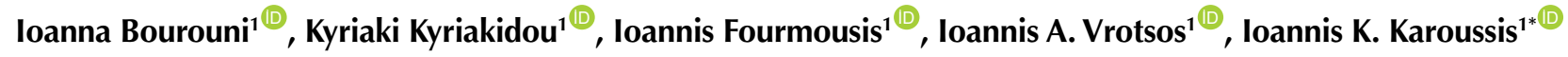 \\ 'Department of Periodontology, School of Dentistry, National and Kapodistrian University of Athens, Thivon 2 str, Goudi, \\ 11527 Athens, Greece
}

\author{
*Correspondence to \\ Ioannis K. Karoussis, \\ Associate Professor, Department \\ of Periodontics, \\ School of Dentistry, \\ National and Kapodistrian \\ University of Athens \\ Thivon 2, 11527, Athens, \\ Greece. \\ Tel: +30 2107461314 \\ Email: ikaroussis@dent.uoa.gr
}

Received: December 12, 2020 Accepted: February 16, 2021 epublished: July 6, 2021

\begin{abstract}
Introduction: Photomodulation is a promising strategy for optimizing tissue healing, but its photomodulatory effects on the synergistic cellular metabolism of gingival and bony tissues remain largely unknown. The aim of the present study was to evaluate the photomodulatory effects of a diode laser $(810 \mathrm{~nm})$ on osteoblasts, HGFs and their co-cultures in vitro.

Methods: Primary cultures of HGFs, cultures of immature osteoblastic cells (MG63) and their cocultures were irradiated with a diode laser $(810 \mathrm{~nm}), 15 \mathrm{~J} / \mathrm{cm}^{2}$. Cell cultures were examined for cellular proliferation (MTT assay), viability (FDA/PI staining) after 24, 48 and 72 hours and cell differentiation (qPCR of collagen type 1a - COL1a and alkaline phosphatase expressions - ALP) after 7 days.

Results: Photomodulation with an 810-nm diode laser increased cell proliferation at all time points. COL1a gene expression increased both in HGF and co-cultures. ALP expression was up-regulated in osteoblastic cultures, but co-cultures with fibroblasts negated this response.

Conclusion: The $810-\mathrm{nm}$ diode laser positively affected cell proliferation and viability in all experimental groups. The statistically significant increased COL1a gene expression at 7 days after irradiation both in the irradiated HGF and co-cultures suggests that low-level laser therapy (LLLT) stimulated extracellular matrix (ECM) formation signaling in both cell types.
\end{abstract}

Keywords: Co-culture; Diode laser; Human gingival fibroblasts; Osteoblasts; Photomodulation; 810 $\mathrm{nm}$.

\section{Introduction}

An 810-nm diode laser is surgically used for the safe elimination of soft oral tissue lesions, and is generally employed at the clinical practice facilitating the treatment of extended areas in a single application, and allowing precise control of laser fluence application within the oral cavity. ${ }^{1}$ There are two types of effects that a lowlevel laser produces; primary and secondary. Primary effects regard vasodilation, lymphatic drainage, cellular activity and metabolism, enhancement of the blood flow, activation of fibroblasts and neutrophils, and increase of the pain threshold. Overall, the secondary effects are the aggregation of prostaglandin, immunoglobulin and lymphocytes and beta-endorphin, in the nervous tissue, infection and inflammation, and therefore, pain, soreness, and the immune response are reduced. ${ }^{2}$

At low output power, applied in a non-contact mode, low-level laser therapy (LLLT, 100-500 mW) offers several advantageous results on the target tissue. ${ }^{3}$ It has been theorized that the radiation of a red or nearinfrared spectrum (600-1000 nm) causes stimulation of mitochondria. The keystone for LLLT outcomes is the ability of light to function as a photo-stimulant in living cells by exerting its effects through photoelectric, photo-physical and photochemical phenomena. In this manner, lasers present healing properties such as anti-inflammatory action, analgesia and tissue healing promotion (photobiomodulation). ${ }^{4,5}$

Despite significant research efforts around the effects of LLLT on various cells or tissues, the appropriate doses of radiation, energy densities, time, and irradiation conditions as well as the appropriate individual settings in the various laser devices, which predictably lead to optimal therapeutic effects, have not been clarified yet. LLLT leads to just a small temperature increase in the treated area, thus avoiding sublimation or thermal lesions. ${ }^{6}$ 
It has been supported that the proper time to apply LLLT with a diode laser $(600-1000 \mathrm{~mm})$ is in the early stages of healing where the potential of cell proliferation increases. Several in vitro and in vivo studies report the effect of LLLT on various cells, but most of them focus primarily on fibroblasts or osteoblasts alone. Specifically, in gingival fibroblasts, several data report increased cell proliferation, increased mRNA production of the growth factors VEGF and TGF- $\beta$ and increased mRNA production of COL1a. In osteoblasts, increased cell proliferation, increased expression of growth factors BMP- 2 and TGF- $\beta 1$, increased alkaline phosphate activity and increased mRNA expression of osteocalcin, bone sialoprotein and osteoprotegerin have been reported. ${ }^{7-10}$

Alkaline phosphatases (ALPs) are expressed by premature osteoblasts and one of their main functions is the promotion of metal deposition by bone marrow cells. ${ }^{11}$ Increased expression of ALP is applied as a reliable index of osteoblast differentiation. ${ }^{12}$

Collagen is the most abundant extracellular matrix (ECM) protein in osseous tissues and represents about $90 \%$ of the organic phase of the ECM. It is characterized by high elasticity and durability, which provides framing at hard tissues. The COLlal gene is expressed primarily by fibroblasts and produces the precursor to the $\alpha 1$ chain of collagen type I, called pre- $\alpha 1$. This chain is combined with not only another pre- $\alpha 1$ chain, but also with a pre- $\alpha 2$ chain (produced by the COL1a2 gene), forming a molecule of pro-collagen type I. This triple helix, processed and cut from enzymes outside the cell, is converted to the mature form of collagen. These mature molecules are then organized into long, thin fibrils that cross-link with another molecule in the space around the cells, resulting in the formation of very strong mature collagen type $1 .{ }^{12}$

Collectively, published data support that LLLT affects tissues, especially when they are in the healing phase. However, whether LLLT is an effective treatment approach has been challenged to date and has led to controversial conclusions. The reason is that the exact mechanism by which this photochemical phenomenon acts has not been fully understood. The great heterogeneity of the studies in terms of wavelengths, output power, time of application, and technical parameters such as the type and diameter of the optical fiber and the distance between the optical fiber and the target cells, as well as the inability to transfer the in vivo conditions to the laboratory, has led to the diversity of the results of the various studies. Moreover, no study up to now has focused on the outcome of LLLT on conditions resembling the interface between bone and soft tissue like in cases of surgical procedures when a mucoperiosteal flap is elevated.

Therefore, the aim of this in vitro study was to assess the effect of LLLT with a diode laser $(810 \mathrm{~nm})$ on the viability, proliferation and differentiation of human premature osteoblasts, human gingival fibroblasts (HGFs) and their co-culture.

\section{Materials and Methods \\ Cell Cultures}

MG63 human osteoblast-like cells (LGC standards, Germany) were grown and maintained in a controlled atmosphere $\left(5 \% \mathrm{CO}_{2}, 37^{\circ} \mathrm{C}\right)$ in growth medium represented by Dulbecco's Modified Eagle's Medium (DMEM Gibco Grand Island, NY) low glucose, 10\% fetal bovine serum (FBS) supplemented with $1 \%$ antibiotics (penicillin-streptomycin, Sigma-Aldrich, Germany) solution. MG63 cells were used between the $3^{\text {rd }}$ and $4^{\text {th }}$ passages seeded at the density of $1 \times 10^{4} \mathrm{cells} / \mathrm{cm}^{2}$ in 12 multiwell plates.

Cell cultures of primary human gingival fibroblasts, HGFs (LGC standards, Germany), were cultured in the DMEM/F12 medium supplemented with 5\% FBS and 1\% penicillin-streptomycin solution in standard conditions, $37^{\circ} \mathrm{C}, 5 \% \mathrm{CO} 2$. HGFs were seeded at the density of $1 \times 10^{4}$ cells $/ \mathrm{cm}^{2}$ in 12 multiwell plates.

\section{Co-cultures}

For the co-cultures of premature osteoblasts and HGFs, in order to provide the necessary nutrients for both cells cultures a mixture of DMEM 10\% FBS, low glucose and DMEM/F12 5\% FBS supplemented with antibiotics streptomycin/penicillin $1 \%$ was used in a ratio of $1: 1 \mathrm{v} / \mathrm{v}$. The seeding cell density of both cell lines was halved in order to maintain the ratio of $1 \times 10^{4}$ cells $/ \mathrm{cm}^{2}$. The cells were seeded in 12-well plates for 24 hours, 48 hours, 72 hours, and 7 days. All the experiments were performed in triplicate in 3 independent runs.

\section{Laser Irradiation}

Twenty-four hours after seeding MG63 cells, HGF and their co-cultures were irradiated by an $810 \mathrm{~nm}$ diode laser (FOX, ARC) via a $300 \mu \mathrm{m}$ optical fiber. The LLLT was applied in concentric circular movements of the optical fiber at a distance of $10 \mathrm{~mm}$ from the surface of the culture in order to irradiate the entire well containing the cells. The irradiation settings selected based on prior experiments of our group (data not shown) were as follows: the power output of $500 \mathrm{~mW}$ and irradiation duration of 120 seconds (Table 1). As control groups were not irradiated, MG63, HGF and their co-cultures, seeded on tissue culture plastic were used.

\section{MTT Assay}

MTT assay (Sigma-Aldrich, Germany) was used to assess the cell proliferation rate. At each time point, the medium from the experimental groups and controls was removed. $200 \mu \mathrm{L}$ of MTT (SIGMA M56655) solution and $1.8 \mathrm{~mL}$ of DMEM without phenol red were added to each well incubated at $37^{\circ} \mathrm{C}$ for 4 hours. After discarding the supernatants, the dark blue formazan crystals were 
Table 1. Specific Diode Laser Settings Applied in the Study

\begin{tabular}{lc}
\hline Parameter & Value \\
\hline Type of laser & Diode \\
Emission mode & Continuous wave \\
Wavelength & $810 \mathrm{~nm}$ \\
Delivery system & Optical fiber $300 \mu \mathrm{m}$ \\
Power & $500 \mathrm{~mW}$ \\
Distance from the surface & $10 \mathrm{~mm}$ \\
Spot diameter at the well & $4.6 \mathrm{~mm}$ \\
Spot area at the well & $0.16 \mathrm{~cm}$ \\
Energy density at the spot area & $300 \mathrm{~J} / \mathrm{cm}$ \\
Energy density at the well & $15 \mathrm{~J} / \mathrm{cm}$ \\
\hline
\end{tabular}

dissolved by adding $2 \mathrm{~mL}$ of solvent $(0.1 \mathrm{~N} \mathrm{HCl}$ in absolute isopropanol) and quantified spectrophotometrically (VersaMax ELISA Microplate Reader, Biocompare, USA) at $570 \mathrm{~nm}$.

\section{Cell Viability}

Cell viability was detected applying the double staining FDA/PI (Sigma-Aldrich, Germany ) method. Briefly, $5 \mu \mathrm{L}$ of dysoxic fluorescein solution and $2 \mu \mathrm{L}$ of iodine propidium were added in $10 \mathrm{ml}$ PBS. One milliliter of the solution was added to each well incubated for 3 minutes at room temperature in a dark and humid place. In the fluorescence microscope (Olympus BX60), the living cells appeared green (FDA stain), while the dead cells were observed as red in color (PI stain).

\section{RNA Extraction and cDNA Synthesis}

RNA extraction from MG63 cells, HGF and their cocultures was performed using NucleoZOL (MachereyNagel) according to the manufacturer's instructions. Following extraction, the RNA was quantified by using a nanodrop photometer (Shimatzu, Japan). Reverse transcription was performed with $1 \mu \mathrm{g}$ of total RNA and ProtoScript II First Strand cDNA Synthesis kit (NEB, US) according to the manufacturer's instructions. cDNA was stored at $-20^{\circ} \mathrm{C}$ until further analysis.

\section{qPCR Analysis}

In order to quantify gene expression, qPCR was performed using SYBR green chemistry and specific primers for the genes COL1a and ALP (Table 2). The analysis was performed in a Bio-Rad iQ5 real-time PCR cycler (BioRad, USA). The expression was normalized to GAPDH (glyceraldehyde 3-phosphate dehydrogenase) and analysis was performed using the delta-delta Ct (DDCt) method for relative quantification. Amplification curves and melt curves were obtained from each sample in order to calculate the cycle threshold $(\mathrm{Ct})$ and verify specific product formation respectively.

\section{Statistical Analysis}

Following the normality test, a $t$ test was performed for the analysis of differences in the cell number between the experimental groups at each time point. Statistically significant differences were set at $P$ value $<0.05$.

\section{Results}

\section{MTT Assay}

The proliferation rate of all the experimental groups statistically significantly increased $(P<0.001)$. A proliferative peak was observed in the irradiated coculture experimental group (Figure 1).

As shown in Figure 2, 48 hours after irradiation, the proliferation of the control groups was in accordance with the characteristics of each cell line. Irradiated HGF showed a higher increase of their population compared to irradiated MG63 and the irradiated co-cultures.

72 hours after irradiation, a statistically significant difference $(P<0.001)$ in cell proliferation for all groups was observed. A pronounced increase of cell population in the co-culture experimental groups was noticed (Figure 3).

\section{Cell Viability}

IMO confirmed the results obtained by the MTT test at all the time points (data for 24 hours and 48 hours not shown). However, in addition to the optimal cell viability, a remarkable distribution of the cells in the co-culture wells, where the niches of the small cells were surrounded by elongated cells (arrows), was revealed (Figure 4f).

\section{Gene Expression}

The ALP gene expression showed a statistically significant increase, $P<0.005$, only in the irradiated MG63 cells (Figure 5).

The COLla gene was overexpressed $(P<0.001)$ both in the irradiated HGF and irradiated co-cultures (Figure 6).

\section{Discussion}

LLLT applications to promote the acceleration of wound healing and reduction of inflammation and pain are well documented. ${ }^{6}$ At a subcellular level, LLLT increases cellular metabolism, the rate of proliferation, and protein expression of biomarkers and growth factors, thus enhancing the healing process.

In the international literature, there are numerous studies that examine the use of diode lasers for LLLT.

Table 2. Primers Sequences Used in the Experiment

\begin{tabular}{ll}
\hline \multirow{2}{*}{ GAPDH } & F:CCTGCACCACCAACTGCTTA \\
& R:GGCCATCCACAGTCTTCTGAG \\
Col1a1 & F:TGCTCGTGGAAATGATGGTG \\
& R:CCTCGCTTTCCTTCCTCTCC \\
\multirow{2}{*}{ VEGF } & F:CTCCTGTCTTGGGTCATTG \\
& R:AGCTGCGCTGATAGACAATCC \\
\hline
\end{tabular}




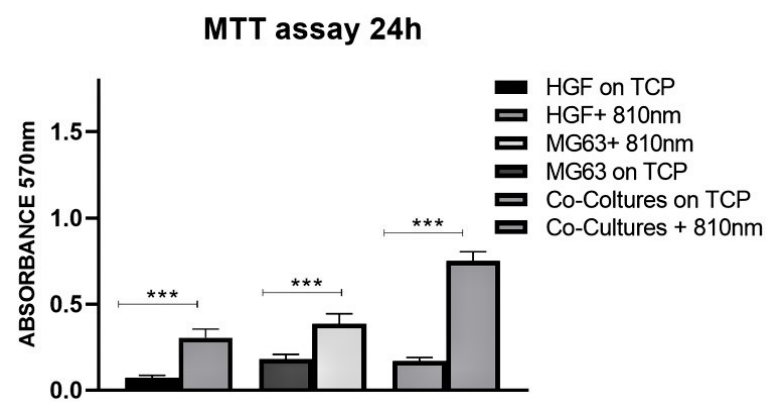

Figure 1. Comparison of Cell Proliferation 24 Hours After Irradiation.

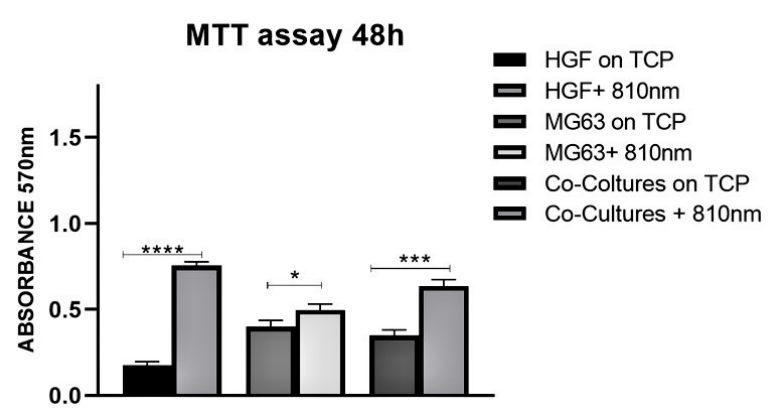

Figure 2. Comparison of Cell Proliferation of Irradiated and Nonirradiated Cells at $48 \mathrm{~h}$.

However, it is a fact that great heterogeneity exists in relation to methodology, wavelengths, frequency and duration of irradiation among published data, which is a matter of concern for the determination of the optimal parameters that should be applied to improve healing processes.

Our group has examined various wavelengths and energy densities for the optimal settings to be used in cell cultures in order to evaluate their outcome in terms of cell proliferation, differentiation and growth factor expression (Data not presented here). The parameters used in the present study have been verified as offering optimal results of LLLT. To our knowledge, this is the first study that investigated the role of LLLT with a wavelength of $810 \mathrm{~nm}$ in the proliferation and differentiation of two different cell populations (HGF, MG63), co-existing in vitro. The selection of this specific co-culture was based on the necessity to resemble a clinical condition arising during flap elevation, where it would be desirable to increase collagen production by gingival fibroblasts, thus promoting the healing of the soft tissue while concomitantly assess the effects of LLLT on a cellular level, as far as the osseous tissue is concerned and at the same time evaluate the effect of LLLT on the concomitantly

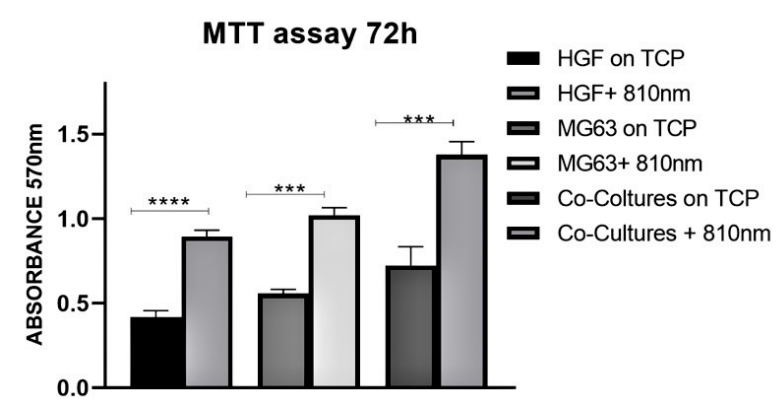

Figure 3. Comparison of Cell Proliferation of Irradiated and Nonirradiated Cultures at $72 \mathrm{~h}$.
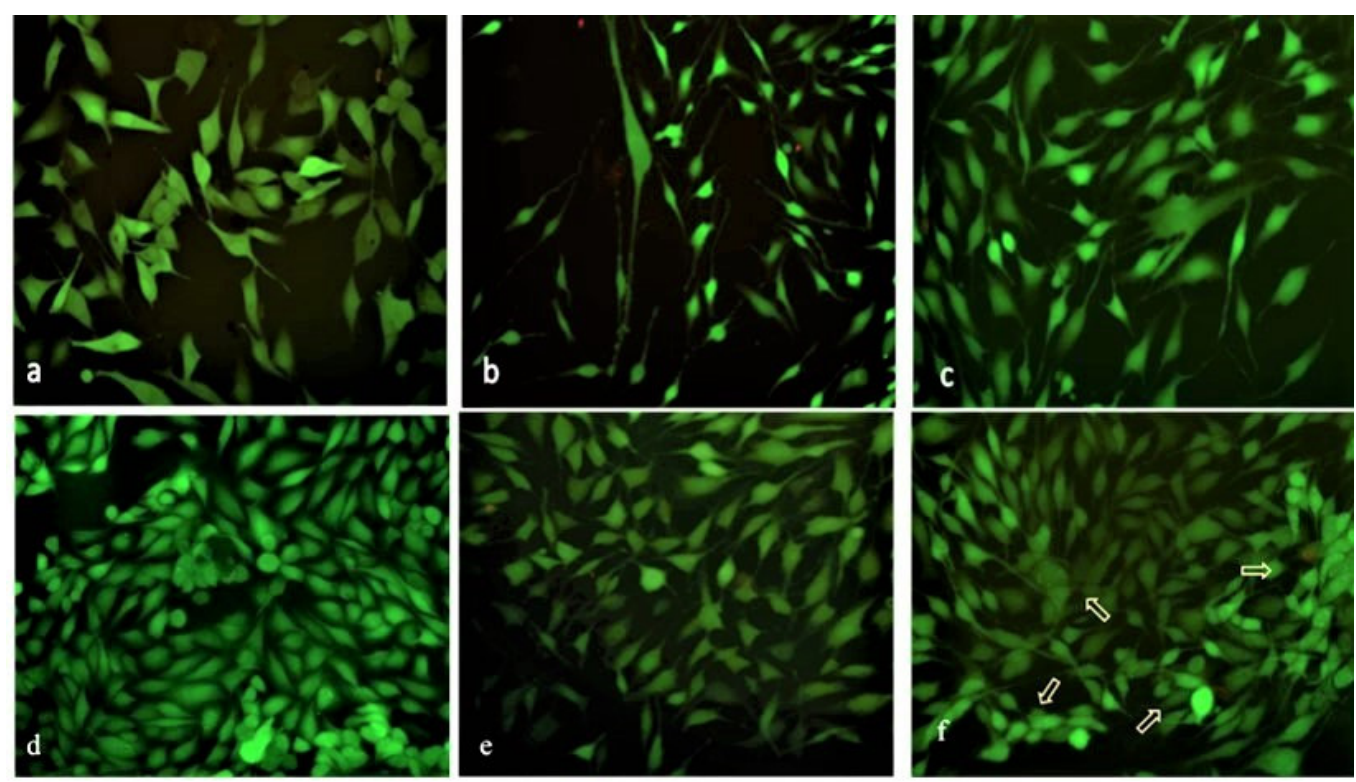

Figure 4. Comparison of Cell Viability of Non-irradiated (a) MG63, (b) HGF, (c) Co-cultures and 810 nm Irradiated, (d) MG63, (e) HGF, and (f) Co-cultures at $72 \mathrm{~h}$. 
ALP GENE EXPRESSION 7 DAYS

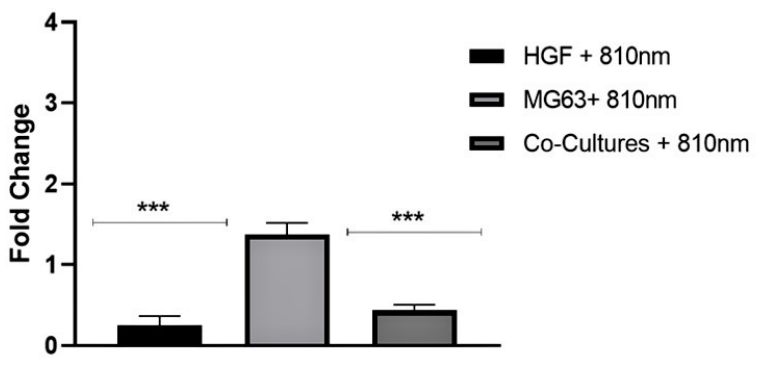

Figure 5. ALP Gene Expression of All Irradiated Experimental Groups in 7 Days.

ALP GENE EXPRESSION 7 DAYS

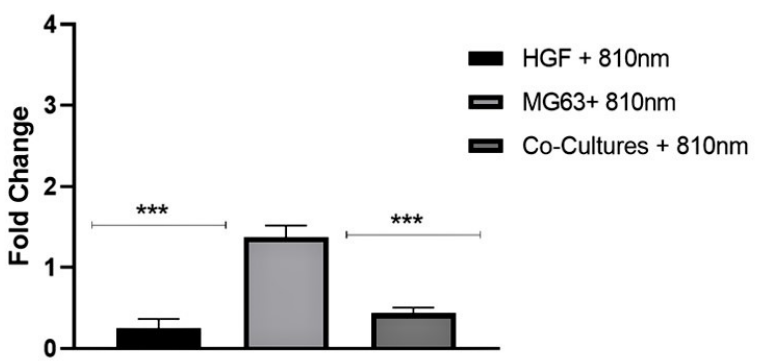

Figure 6. COL1a Gene Expression for All Irradiated Experimental Groups in 7 Days.

irradiated bony tissue.

The increased proliferation rate of HGF at 24, 48 and 72 hours found in our study is in accordance with previous research data that concluded on a statistically significant proliferation increase of the same cells at 24 and 48 hours after laser irradiation with similar wavelengths. ${ }^{13,14}$

Moreover, our results are supported by a systematic review ${ }^{15}$ based on 13 experimental studies, where it was underlined that further research is anticipated to provide sound evidence on the best settings and application protocols.

In the present study, the proliferation rate of osteoblasts at 24, 48 and 72 hours was found statistically significantly increased in the irradiated groups. The amplification of the proliferation rate of osteoblastic cells after LLLT has been documented by our group in a previous publication. ${ }^{16}$ The increased proliferation rate of irradiated osteoblasts, found in our study, is in accordance with previous research data on the outcome of LLLT using $810 \mathrm{~nm}$ by Soleimani et al who concluded on a statistically significant increase of proliferation. ${ }^{17}$ Furthermore, our results verify the results of the later study in terms of ALP expression, 7 days after irradiation. Both results of the studies are in accordance with those of an experimental model applying LLLT with an 810 -nm diode in murine osteoblasts. ${ }^{18}$ It should be mentioned that Chang et $\mathrm{a}^{18}$ found $810 \mathrm{~nm}$ to be superior to other wavelengths evaluated.
However, the lack of ALP expression in the co-cultures in our study is explored for the first time in the literature. A possible explanation is that ALP gene down-regulation in the co-cultures indicates a laser-induced proliferative boost of osteoblastic cells under the interaction with HGF. This speculation is justified by the fact that our mixed population presented a substantial proliferative activity up to 3 days after irradiation (Figure 3). However, there is a chance that a ceiling effect occurred at the 72-hour time point in terms of proliferation; thus, ALP expression may have diminished. This could be due to cytokine signalling of fibroblasts that may have altered the differentiation capacity of osteoblastic cells in the co-culture. Further investigation into the proliferation rates and immunohistochemical characterization of the proportion of the cells in this co-culture for up to 7 days would be interesting.

The most important finding of this study was revealed by the gene expression of the experimental groups in relation to the COL1a gene. This is the first study to evaluate the influence of LLLT with an 810-nm diode on the expression of COLla by HGFs. Interestingly, a statistically significant increase of this gene expression was revealed in both irradiated HGF and co-cultures, leading to a logical conclusion of the utilization of the examined wavelength with the applied parameters in clinical conditions in a further scientific project. Perhaps the utilization of a single LLLT after the elevation of a mucoperiosteal flap with the suggested parameters would promote post-surgical healing of the operated area.

\section{Conclusion}

Photomodulation accelerated the cell proliferation of both cell types examined as well as their co-culture. Gene COL1a overexpression was induced by LLLT with an 810nm diode.

It could be speculated that during flap surgery, single low-level laser irradiation using $810 \mathrm{~nm}$ would promote healing of the soft tissues since collagen production is significantly increased by HGF.

Further studies should be performed to clarify the cellular interactions between HGFs and osteoblasts in order to improve the clinical outcome of LLLT.

\section{Conflict of Interests}

The authors certify that they have no commercial or associative interest that represents a conflict of interest or funding in connection with the manuscript.

\section{References}

1. Akbulut N, Kursun ES, Tumer MK, Kamburoglu K, Gulsen U. Is the $810-\mathrm{nm}$ diode laser the best choice in oral soft tissue therapy? Eur J Dent. 2013;7(2):207-11. doi:10.4103/1305-7456.110174

2. Luke AM, Mathew S, Altawash MM, Madan BM. Lasers: A Review with Their Applications in Oral Medicine. J Lasers 
Med Sci. 2019;10(4):324-9. doi:10.15171/jlms.2019.52

3. Ishikawa T, Hagiwara K, Ochiya T. Generation and hepatic differentiation of human iPS cells. Methods Mol Biol. 2012; 826:103-14. doi:10.1007/978-1-61779-468-1_9

4. Carroll L, Humphreys TR. LASER-tissue interactions. Clin Dermatol. 2006;24(1):2-7. doi:10.1016/j. clindermatol.2005.10.019

5. Gkogkos AS, Karoussis IK, Prevezanos ID, Marcopoulou KE, Kyriakidou K, Vrotsos IA. Effect of Nd:YAG Low Level Laser Therapy on Human Gingival Fibroblasts. Int J Dent. 2015;2015:25894. doi:10.1155/2015/258941

6. Huang YY, Chen AC, Carroll JD, Hamblin MR. Biphasic dose response in low level light therapy. Dose Response. 2009;7(4):358-83. doi:10.2203/dose-response.09-027

7. Brignardello-Petersen R, Carrasco-Labra A, Yanine N, Ulloa C, Araya I, Pintor F, et al. Positive association between conflicts of interest and reporting of positive results in randomized clinical trials in dentistry. J Am Dent Assoc (1939). 2013;144(10):1165-70. doi:10.14219/jada. archive.2013.0035

8. Almeida-Lopes L, Rigau J, Zângaro RA, Guidugli-Neto J, Jaeger MM. Comparison of the low level laser therapy effects on cultured human gingival fibroblasts proliferation using different irradiance and same fluence. Lasers Surg Med. 2001;29(2):179-84. doi:10.1002/lsm.1107

9. Poon VK, Huang L, Burd A. Biostimulation of dermal fibroblast by sublethal Q-switched Nd:YAG 532 nm laser: collagen remodeling and pigmentation. $J$ Photochem Photobiol B. 2005;81(1):1-8. doi:10.1016/j. jphotobiol.2005.05.006

10. Millán JL. Alkaline Phosphatases: Structure, substrate specificity and functional relatedness to other members of a large superfamily of enzymes. Purinergic Signal. 2006;2(2):335-41. doi:10.1007/s11302-005-5435-6

11. Zhang L, Buchet R, Azzar G. Distinct structure and activity recoveries reveal differences in metal binding between mammalian and Escherichia coli alkaline phosphatases.
Biochem J. 2005;392(Pt 2):407-15. doi:10.1042/bj20050509

12. Heinemann DE, Siggelkow H, Ponce LM, Viereck V, Wiese KG, Peters JH. Alkaline phosphatase expression during monocyte differentiation. Overlapping markers as a link between monocytic cells, dendritic cells, osteoclasts and osteoblasts. Immunobiology. 2000;202(1):68-81. doi:10.1016/s0171-2985(00)80054-6

13. Kreisler $M$, Meyer C, Stender E, Daubländer $M$, Willershausen-Zönnchen B, d'Hoedt B. Effect of diode laser irradiation on the attachment rate of periodontal ligament cells: an in vitro study. J Periodontol. 2001;72(10):1312-7. doi:10.1902/jop.2001.72.10.1312

14. Azaripour A, Azaripour M, Willershausen I, Noorden C, Willershausen B. Photodynamic Therapy has no Adverse Effects in Vitro on Human Gingival Fibroblasts and Osteoblasts. Clin Lab. 2018;64(7):1225-31. doi:10.7754/ Clin.Lab.2018.180220

15. Ren C, McGrath C, Jin L, Zhang C, Yang Y. Effect of diode low-level lasers on fibroblasts derived from human periodontal tissue: a systematic review of in vitro studies. Lasers Med Sci. 2016;31(7):1493-510. doi:10.1007/s10103016-2026-4

16. Karoussis IK, Kyriakidou K, Psarros C, Lang NP, Vrotsos IA. Nd:YAG laser radiation $(1.064 \mathrm{~nm})$ accelerates differentiation of osteoblasts to osteocytes on smooth and rough titanium surfaces in vitro. Clin Oral Implants Res. 2017;28(7):785-90. doi:10.1111/clr.12882

17. Soleimani M, Abbasnia E, Fathi M, Sahraei H, Fathi Y, Kaka G. The effects of low-level laser irradiation on differentiation and proliferation of human bone marrow mesenchymal stem cells into neurons and osteoblasts-an in vitro study. Lasers Med Sci. 2012;27(2):423-30. doi:10.1007/s10103-011-0930-1

18. Chang B, Qiu H, Zhao H, Yang X, Wang Y, Ji T, et al. The Effects of Photobiomodulation on MC3T3-E1 Cells via $630 \mathrm{~nm}$ and $810 \mathrm{~nm}$ Light-Emitting Diode. Med Sci Monit. 2019;25:8744-52. doi:10.12659/msm.920396 